Mark J. Benninghoff*, Peter Isfort, Martin Baumann

\title{
How to Support Students in Maximizing their Outcome with Online Cooperation?
}

\begin{abstract}
During the last year, student cooperation has shifted to the digital space. Chats, videoconferencing and online collaboration tools replace physical meetings and face-to-face interactions. While many online tools provide sophisticated technologies to share screens and co-edit manuscripts, the support of collaborative thinking, learning and even fooling around is still underdeveloped, but remains a valuable resource.

This study traces whether the given level of instruction details influences the collaboration effectiveness among student working groups in a practical course in Biomedical Engineering.

Within the semester's practical course of Introduction into Medicine, participating students were divided into three groups (A, B and C) of each 4 to 6 students and had to collaborate on a clinical case. Groups A received detailed information and rules on online collaboration, B less detailed information and $\mathrm{C}$ only basic technical information. Each group was asked to fulfil an additional pre- and post-task before and after the semester's practical course. These tasks consisted of solving combinatory puzzles based on isolated information bits and hints that were distributed among the group members. The groups differed only in the instructions they received to fulfil the task. During their work phases, all groups were observed. The study was conducted twice with different student cohorts.
\end{abstract}

School grades were assigned representing 1 for the best up to 6 for the worst performance. The following averaged results were found: Groups B received the best

*Corresponding author: Mark J. Benninghoff, Institute for Applied Medical Engineering, Helmholtz-Institute, RWTH Aachen University Hospital and RWTH Aachen University, Aachen, Germany, benninghoff@ame.rwth-aachen.de

Peter Isfort, Clinic for Diagnostic and Interventional Radiology, RWTH Aachen University Hospital and RWTH Aachen University, Aachen, Germany

Martin Baumann, Institute for Applied Medical Engineering, Helmholtz-Institute, RWTH Aachen University Hospital and RWTH Aachen University, Aachen, Germany grades (average 1,5 for winter term and average 1,6 for summer term). Second best performance was shown by the groups A (average 2,14 for winter term and average 1,75 for summer term) and finally groups $\mathrm{C}$ performed worst (average 2.10 for winter term and average 1,85 for summer term). In the taken times to solve the puzzle tasks, groups B showed up to be best in the summer term and groups A in the winter term. In all categories, groups $\mathrm{C}$ delivered the worst grades in average.

The results of this study support that it is most reasonable to aid student online collaboration by providing detailed instructions or at least a few hints and instruct them to create their own rules for online collaboration. The results further support that the cooperation gets worse without suggesting any rules or instructions and that student age possesses a negligible influence.

Keywords: online collaboration, practical course, virtual coworking, cooperative working level

https://doi.org/10.1515/cdbme-2021-2066

\section{Introduction}

Cooperative working, especially in interdisciplinary working fields such as (Bio-)Medical Engineering plays an important role in professional daily working routines. However, these routines and the collaboration methods have begun to shift in the last years, and still will develop further in the future [1][2]. Since interdisciplinary collaboration remains notoriously challenging, it proves essential to understand student approaches and potential paradigmatic shifts and to identify innovative gamechangers.

Due to the teaching and learning restrictions following the COVID pandemic, every-day student working routines shifted from one day to the next to the virtual space. Since this also accounts for student learning, we conducted a study to find out main parameters which support or prevent student online cooperation. In this communication, we present and discuss the acquired quantitative and 
qualitative data which may prove useful to boost student cooperative working interaction in the future. The identified features and boundary conditions can successfully and broadly be implemented across disciplines and provide innovative support in this new virtual space.

The Institute of Applied Medical Engineering at the Helmholtz-Institute, RWTH Aachen University, provides an innovative practical course named "Introduction into Medicine" in which the students mainly work in small groups of 4 to 6 participants on an interdisciplinary medical and clinical topic. Each team is asked to consent on a selfselected clinical problem and frames a specific solution in a cooperative approach. Groups are given time to elaborate hypotheses on the clinical problem, do literature research, receive professional advice from clinicians and finally summarize the problem and possible solutions in an essay. Due to the pandemic, group work was only possible via videoconferencing. In order to straighten the student work, we conducted this study to find out which level of preceding instruction proves best to support students in online collaboration.

\section{Methods and Materials}

In the summer semester 2020 and winter semester 2020/21, student participants of the practical course were randomly assigned to working groups. Each of the 32 groups consisted of 4 to 6 participants and belonged to one of three test groups:

- Test group A with a total of 11 student groups

- $\quad$ Test group B with a total of 11 student groups

- Test group C with a total of 10 student groups

Group members were not pre-tested with behalf on collaboration experience or logical capacity.

Each of the 3 test groups received an individual briefing document, explaining the objective and virtual platform specifics in a graded detailed approach from highly detailed (group A) to only basic (group C) information:

Group A received the most information, detailed notes, rules and instructions for effective cooperation which were compiled on 2 written pages. These included definitions for different facilitator roles (e.g. moderator, time keeper), group tasks (e.g. working with topics and notes), special rules for online meetings and general advices on how to respect all participants' contributions and ideas. In addition, advices for the facilitator roles were added on how to prepare and conduct the respective group meetings.
Group B received a less detailed instructional set. They were given the instruction to create their own working rules and were asked to find a consent on how to work respectfully. This included only 2 guiding principles to aid them: Firstly to consider what are the differences compared to an offline meeting and which rules they want to establish to compensate for these differences. Secondly, the groups were asked to agree on facilitator meeting roles and to define associated tasks respectively.

Group C was only provided with basic technical information on how to get in contact to the other student members of their respective working group.

Group agency requires joint attention, coordinated action and intersubjective intentionality [3]. In order to measure the efficiency of the groups' online collaboration, each working group was asked to solve a combinatory puzzle at the beginning of the respective semester P1, and a similar puzzle at the end of the semester P2 (detailed puzzle description: see next chapter). Times to complete the puzzles were measured. In each puzzle, every single member of a working group received a separate chunk of information consisting of two to three bits of isolated information that were not understandable by themselves, but only after the information of the whole group was assembled semantically (see Figure 1 top for examples). Every single information bit referred crosswise to all group members' bits. Additional information had to be gained from internet data bases.
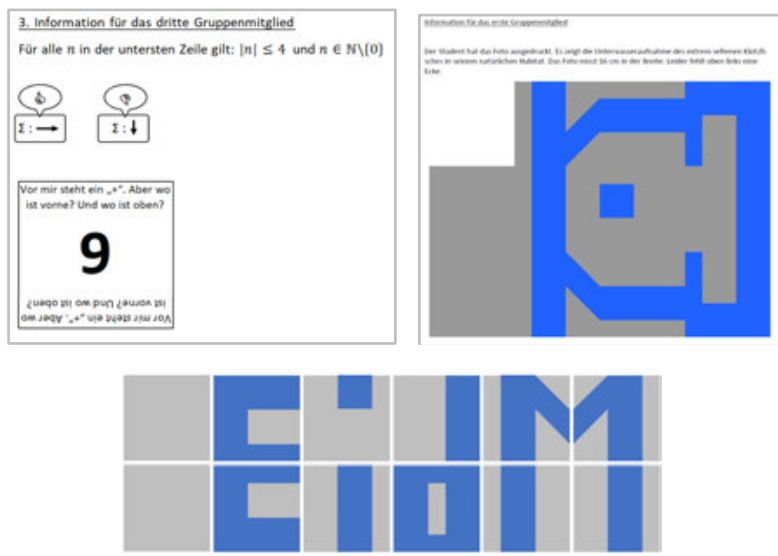

Figure 1: Top left: typical information chunk of $P 1$ with three information bits. Top right: typical information chunk of $\mathrm{P} 2$. Bottom: solution of P2 after all tiles had been rearranged after contribution of each single group member.

All working groups were evaluated during their working time by an external observer, who documented the quality of cooperation and performance. A school grade was granted from the overall impression rate by 
considering interactivity, documentation, sharing findings, delegation and execution. In total, 25 surveillance parameters, noted by the observing persons, contributed to each grade. Grades ranged from 1 for the best up to 6 for the worst performance

All groups were randomly mixed with respect to gender (68\% male and 32\% female students).

Zoom (Zoom Inc., San José, California) was used for all sessions and every group was provided with a working licence. Observations were done by hidden and nonparticipating persons, who noted collaboration data e.g number of interactions, collaboration stringency and time to success.

Written informed consent was obtained from all students prior to the beginning of the respective practical course. No participant withdrew his or her consent.

\subsection{The Puzzle Tasks}

In the P1 puzzle, every group's task was to reconstruct a three digits code out of 10 given digits. The P2 puzzle task was to rearrange 10 square tiles with geometric colouring to form a readable letter code, see Figure 1 bottom). In order to increase motivation, both P1 and P2 were integrated in a small appealing plot.

Both puzzles' solutions were visible only after all information bits from the entire working group were interactively combined and correctly assembled.

\section{Results}

In average, the working groups had 4,5 meetings with an average duration of 1 hour and 27 minutes. There were no significant differences between the three test groups in number of meetings or in the respective meeting durations. Also, meetings with and without a moderator were distributed equally among all test groups.

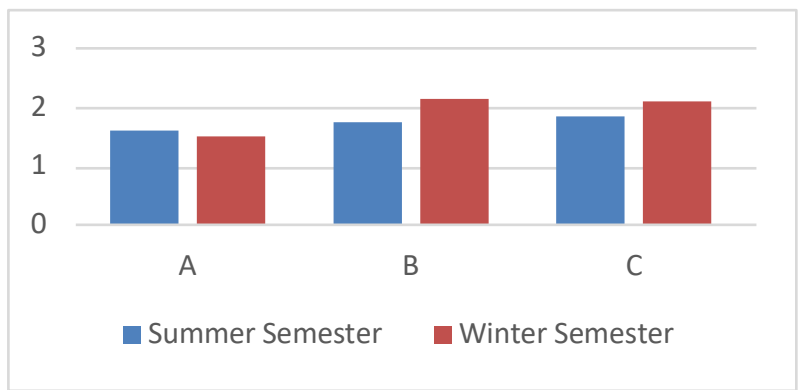

Figure 2: Achieved average school grades sorted after test groups and semester.

\subsection{School Grades}

The documented school grades showed only small differences between the test Groups. These differences can be only considered as a trend but actually there were no significant findings (Figure 2).

\subsection{Solutions of the Puzzle Tasks}

The difference in time which was needed to finalize the puzzle tasks showed to be significantly different between the test groups A and B vs. C and thus between the respective settings (Figure 3 ). It is noticeable that group $\mathrm{C}$ took the longest to complete the task. If the data from both semesters are combined to be more statistically meaningful, one can see that the $\mathrm{A}$ and $\mathrm{B}$ groups range almost at an identical level (Figure 4) and groups $\mathrm{C}$ needed in average 17 minutes more time to solve, which means an increase of about $40 \%$ in comparison to $\mathrm{A}$ and $\mathrm{B}$.

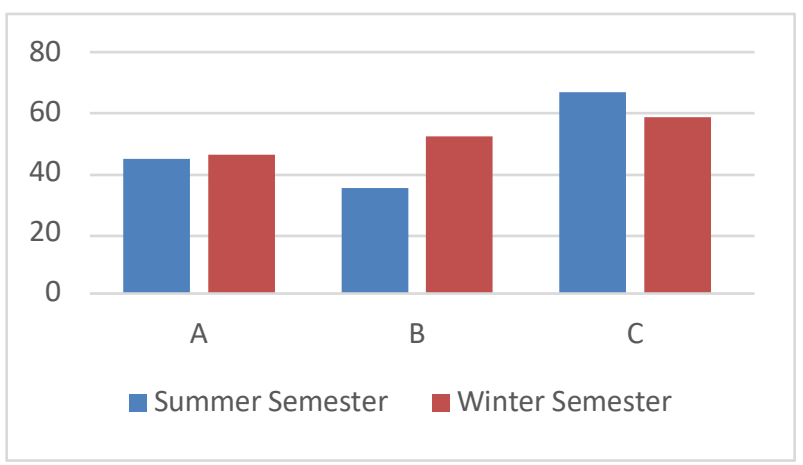

Figure 3: Durations (in minutes) to solve the respective puzzle task, sorted after test groups and semester.

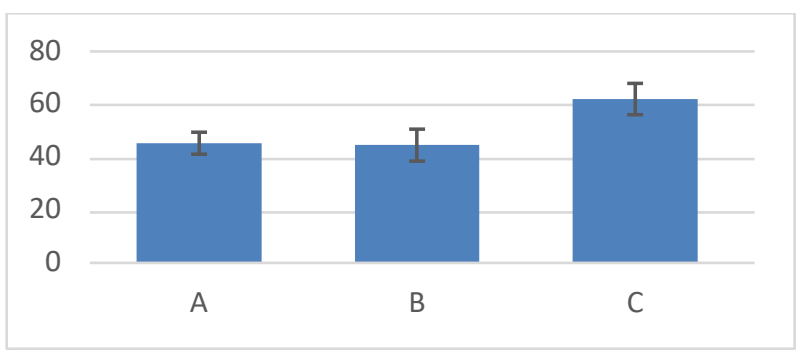

Figure 4: Compiled average durations (in minutes) to solve of both semesters for all test groups.

Whereas the semester has no visible effect on the time to solve the puzzles (Figure 3), there exists a correlation between the semester and the grade, stating that the summer 
semester student showed a better collaboration quality and thus received the better grades (Figure 2).

The observations showed that the number of active contributions within the meetings is higher when a moderator guides the meeting. This correlation is significant. No correlation was found between the subject of study and the time to solve the puzzle or the grade, respectively.

\section{Discussion}

The results demonstrate that there is a significant difference in required time for the puzzle between the different test groups. Test groups which received major (A) or minor detailed (B) instructions needed in average 17 minutes less time to solve the puzzle than test group $\mathrm{C}$, with no information on how to collaborate efficiently.

For the student target groups, the semester correlates with the students' age. In this study, summer semester students were older than winter semester students. The findings shown in Figure 2 thus suggest that student age may also play a role in the quality of student online collaboration, since summer students performed better with respect to the grades.

Our initial apprehension that highly detailed working instructions would lead to an increase in time to solve the puzzles due to an increased care for the instructions themselves was unfounded (Figure 4).

\section{Conclusions}

The results of this study support that it is most reasonable to aid student online collaboration by providing them with a manual that informs about basic online collaboration rules and instruct them to create their own rules for online collaboration. As long as this information is provided, it almost plays no role, how detailed it is given.

The results further support that the cooperation gets worse without suggesting any rules or instructions and that student at higher level have no critical benefit. Future research might be needed to establish the findings in the different areas for interactivity and relationship building, communication and documentation, strategic thinking and analytics, delegation and execution.

In general, the insight from this broad data set on a cooperative working attitude have already led to a paradigm shift in our teaching routines, which now incorporate a set of basic instructions for online collaboration. As the target students in this study stem from engineering and nature sciences, we believe that the findings will benefit students of multiple disciplines.

\section{References}

[1] Fitzgerald DA, Jones N, Choudhury S, Friedner M, Levin N, Lloyd S, Meyers T, Myers N \& Raikhel E (2014) The collaborative turn: Interdisciplinarity across the human sciences. In Somatosphere, Available at: http://somatosphere.net/2014/the-collaborative-turninterdisciplinarity-across-the-human-sciences.html/

[2] Josef B, Back A (2018) (2018): Coworking as a New Innovation Scenario from the Perspective of Mature Organisations, In: Tipurić, Darko Labaš, Davor (Ed.): 6th International OFEL Conference on Governance, Management and Entrepreneurship. New Business Models and Institutional Entrepreneurs: Leading Disruptive Change, pp. 491-507

[3] Stahl, G., Law, N., Cress, U. et al. Analyzing roles of individuals in small-group collaboration processes. Intern. J. Comput.-Support. Collab. Learn. 9, 365-370 (2014). https://doi.org/10.1007/s11412-014-9204-9

\section{Author Statement}

Research funding: The author state no funding involved. Conflict of interest: Authors state no conflict of interest. Informed consent: Informed consent has been obtained from all individuals included in this study. Ethical approval: The research related to human use complies with all the relevant national regulations, institutional policies and was performed in accordance with the tenets of the Helsinki Declaration, and has been approved by the authors' institutional review board or equivalent committee.

This work was supported by the Stifterverband für die Deutsche Wissenschaft within the program Fellowships für Innovationen in der digitalen Hochschullehre to fellows P. Isfort and M. Baumann 